\title{
Avaliação dos fatores de risco para osteoporose e aplicação da ferramenta FRAX clínico em idoso
}

\author{
Evaluation of risk factors for osteoporosis and application of the clinical FRAX \\ tool in the elderly
}

José Seabra Alves Neto ${ }^{1}$ (D) Amanda Raquel Costa Cruz $^{1}$ (D), Francisco de Assis Pereira ${ }^{1,2}$ (D)

\begin{abstract}
RESUMO
A osteoporose é caracterizada pela fragilidade óssea e mudanças em sua microarquitetura. O seu principal desfecho clínico é a fratura por baixo impacto. Uma das principais ferramentas de avaliação clínica do risco de fratura osteoporótica é o FRAX. Este trabalho objetiva avaliar os fatores de risco para osteoporose e a indicação de tratamento em idosos. Foi realizado um estudo transversal, descritivo, com abordagem quantitativa e qualitativa, por meio de um questionário, com 145 pacientes que foram atendidos no setor ambulatorial do DeCos Day Hospital, no período de novembro de 2017 a abril de 2018. Observou-se que a osteoporose prevaleceu no sexo feminino, sedentários e com idade média de 71 anos. A presença de fratura predominou na faixa etária de 70 a 79 anos e nenhum participante fazia tratamento específico para a osteoporose. Conclui-se que orientações dietéticas saudáveis são importantes como medida preventiva e a ferramenta FRAX poderia ser utilizada para estimar a probabilidade de fratura, visto que a densitometria não é a acessível a toda a população.
\end{abstract}

Palavras-Chave: Osteoporose; Fatores de Risco; Fraturas.

\begin{abstract}
Osteoporosis is characterized by bone fragility and changes in its microarchitecture. Its main clinical outcome is a low impact fracture. One of the main tools for clinical assessment of the risk of osteoporotic fracture is FRAX. This work aims to evaluate the risk factors for osteoporosis and the indication for treatment in the elderly. A cross-sectional, descriptive study with a quantitative and qualitative approach was carried out, using a questionnaire, with 145 patients who were assisted in the outpatient sector of the Hospital de Dia DeCos, from November 2017 to April 2018. Osteoporosis prevailed in women, sedentary, and with an average age of 71 years old. The presence of fractures predominated in the age group from 70 to 79 years old, and no participant used specific treatment for osteoporosis. It is concluded that dietary guidelines are important as a preventive measure, and a FRAX tool can be used to estimate the probability of fracture since densitometry is not accessible to the entire population.
\end{abstract}

Keywords: Osteoporosis; Risk Factors; Fractures.

1. Universidade Tiradentes, Aracaju (SE), Brasil.

2. Universidade Federal de Sergipe (UFS), Aracaju (SE), Brasil.

$\square$ José Seabra Alves Neto. Universidade Tiradentes. Avenida Murilo Dantas, número 300, Bairro Farolândia. CEP: 49032-490. Aracaju (SE), Brasil. seabraneto96@gmail.com | Recebido em: 20/05/2020 | Aprovado em: 13/07/2020 


\section{INTRODUÇÃO}

A osteoporose é uma doença que possui como característica a fragilidade óssea e alterações em sua microarquitetura. O seu principal desfecho clínico é a fratura por baixo impacto ${ }^{1}$. Nos Estados Unidos, a doença afeta aproximadamente 25 milhões de pessoas. No Brasil, estudos realizados em Recife mostraram uma prevalência de osteoporose de $28,8 \% \%^{2}$. Atualmente é considerada um dos principais problemas de saúde pública em países desenvolvidos ${ }^{3}$. Além disso, é previsto uma elevação significativa do impacto da osteoporose nos próximos anos devido ao aumento da expectativa de vida populacional ${ }^{4}$.

As fraturas osteoporóticas estão associadas a elevadas taxas de morbidade e mortalidade. São mais prevalentes no sexo feminino e aumentam com a idade ${ }^{5}$. As fraturas podem ser seguidas de recuperação total, dor crônica, incapacidade e morte ${ }^{6}$. Os principais sítios de acometimento são extremidades distais dos rádios, das vértebras, da região proximal do fêmur e da região proximal do úmero ${ }^{7}$.

Existem fatores que se associam com maior valor preditivo para risco de fratura, como: idade; baixo peso ou baixo índice de massa corporal ou perda de peso; fratura osteoporótica prévia; uso de glicocorticoide (dose superior a $5 \mathrm{mg}$ de prednisona/dia ou equivalente por período igual ou superior a três meses); uso de alguns anticonvulsivantes (relação com o metabolismo da vitamina D); sedentarismo; hiperparatireoidismo primário; anorexia nervosa; gastrectomia; anemia perniciosa; e hipogonadismo ${ }^{8,9}$.

A densitometria óssea (DXA) é a técnica padrão-ouro utilizada para o diagnóstico de osteoporose. Entretanto, a DXA apresenta limitações, no que se refere que a realização deste exame não é facilmente acessível em todos os países, bem como não é considerado em sua interpretação fatores de risco clínicos que contribuem para piora da qualidade do tecido ósseo e consequentemente maior risco para fratura ${ }^{10}$.

Uma das principais ferramentas de avaliação do risco de fratura osteoporótica é o FRAX (Fracture Risk Assessment Tool). Esta foi desenvolvida em parceria da World Health Organization Collaborating Center for Metabolic Bone Diseases e a Universidade de Sheffield na Inglaterra e foi lançada em 2008. Avalia individualmente cada paciente e associa os fatores de riscos para a osteoporose com a DMO do fêmur medida pela DXA. A partir daí, calcula o risco de fratura de fêmur e de outros sítios nos próximos 10 anos $^{11,12}$. Tendo em vista essas informações, o objetivo do presente estudo foi avaliar os fatores de risco para osteoporose e a indicação de tratamento em idosos por meio da ferramenta FRAX clínico.

\section{MATERIAL E MÉTODOS}

Trata-se de um estudo transversal, descritivo, com abordagem quantitativa e qualitativa, baseado em dados obtidos através de um formulário preenchido durante atendimento ambulatorial, em Hospital privado da cidade de Aracaju, Sergipe.

Os pacientes foram selecionados de acordo com os critérios de inclusão: idade igual ou superior a 65 anos de ambos os sexos, com condições clínicas e cognitivas que permitissem responder ao questionário e que concordaram em participar da pesquisa.

As variáveis consideradas no formulário foram idade, sexo, raça, peso, altura, índice de massa corporal, fatores de risco para osteoporose (sedentarismo, uso de corticoide, menopausa precoce, tabagismo, etilismo, fratura de quadril em pais, síndrome de má absorção e uso de anticonvulsivantes), episódio anterior de queda, risco de fratura e tratamento.

Este estudo foi aprovado pelo Comitê de Ética e Pesquisa da Universidade Tiradentes (CAAE No 73275417.8.0000.5371), parecer número: 2.391.824. Para a realização do estudo, foi previamente obtido dos participantes a assinatura do Termo de Consentimento Livre e Esclarecido (TCLE).

A análise estatística dos dados obtidos foi feita através do programa GraphPad Prism, versão 7.0 para Windows, 2016 (San Diego, CA, USA). Foram empregados o teste Exato de Fisher, o teste Mann Whitney e o teste Qui-Quadrado.

\section{RESULTADOS}

A casuística do estudo foi composta por 145 idosos com média de idade de $71 \pm 6,6$ anos. Em 
relação ao sexo, 102 (70\%) participantes eram mulheres e, $78(53,8 \%)$ eram da raça branca. A média de peso foi de $70,0 \pm 14,0 \mathrm{~kg}$. A média de altura foi de 1,59 $\pm 0,1 \mathrm{~m}$. Os dados demográficos dos participantes do estudo estão apresentados na Tabela 1. Quando estratificados os pacientes por faixa etária foi evidenciado $70(48,3 \%)$ na faixa etária de 65 a 69 anos; $53(36,5 \%)$ na faixa etária de 70 a 79 anos, e $22(15,2 \%)$ na faixa etária de 80 a 90 anos.

\section{Tabela 1}

Dados demográficos dos participantes do estudo. $(n=145)$

\begin{tabular}{lccc}
\hline & Média \pm DP & Mínimo & Máximo \\
\hline IDADE (a) & $71,0 \pm 6,6$ & 65 & 90 \\
SEXO (M/F) & $43 / 102$ & - & - \\
RAÇA (B/NB) & $78 / 67$ & - & - \\
PESO (kg) & $70,0 \pm 14,0$ & 43 & 115 \\
ALTURA (m) & $1,59 \pm 0,1$ & 1,40 & 1,90 \\
IMC (kg/m $\left.{ }^{2}\right)$ & $27,5 \pm 4,8$ & 17,3 & 44,27
\end{tabular}

DP: desvio-padrão; a: anos; M: masculino; F: feminino; B: brancos; NB: não brancos; kg: quilograma; m: metro; IMC: Índice de Massa Corporal

Os fatores de risco para osteoporose que apresentaram maior frequência em nossa amostra foram sedentarismo $(53,1 \%)$, uso de corticoide $(18,6 \%)$, menopausa precoce $(15,9 \%)$, tabagismo $(11 \%)$, etilismo $(3,4 \%)$, fratura de quadril em pais $(1,4 \%)$, síndrome de má absorção $(1,4 \%)$ e uso de anticonvulsivante $(1,4 \%)$. Destes, os que são avaliados na ferramenta FRAX são: história familiar de osteoporose, fratura de quadril em pais, tabagismo, uso de corticoide e etilismo.

Dos 145 participantes do estudo, somente $31(21,4 \%)$ referiram ter o diagnóstico de osteoporose, sendo 29 do sexo feminino e dois do sexo masculino. Destes, $21(67,7 \%)$ relataram sedentarismo, $12(38,7 \%)$ faziam uso de corticoide, sete $(22,6 \%)$ apresentaram menopausa precoce $(<45$ anos), dois $(6,4 \%)$ tabagistas, um $(3,2 \%)$ com síndrome de má absorção, um $(3,2 \%)$ em uso de anticonvulsivante e nenhum etilista, conforme dados da Tabela 2. Entre os 31 pacientes com diagnóstico de osteoporose, nenhum fazia uso de tratamento específico para osteoporose.

\section{Tabela 2}

Distribuição dos fatores de risco para osteoporose entre os portadores de osteoporose. $(n=31)$

\begin{tabular}{lc}
\hline Fatores de risco para osteoporose & $\mathbf{n}(\%)$ \\
\hline Sedentarismo & $21(67,7)$ \\
Uso de corticoide & $12(38,7)$ \\
Menopausa Precoce (<45 anos) & $7(22,6)$ \\
Tabagismo & $2(6,4)$ \\
Fratura de quadril em pais & $2(6,4)$ \\
Síndrome de má absorção & $1(3,2)$ \\
Uso de anticonvulsivante & $1(3,2)$ \\
Etilismo & $0(0)$ \\
\hline
\end{tabular}

n: número, \%: porcentagem

Em relação ao exame de DXA, 86 (59,3\%) relataram já ter sido submetido a sua realização, sendo $74(86 \%)$ do sexo feminino e $12(14 \%)$ do sexo masculino. Dentre os pacientes com diagnóstico de osteoporose por densitometria, as mulheres apresentaram maior prevalência.

Em relação à queda, $46(31,7 \%)$ pacientes relataram já ter apresentado pelo menos um episódio e os indivíduos de raça branca foram os mais acometidos $(58,7 \%)$. A faixa etária com maior taxa de queda foi de 80 a 90 anos e, quando avaliamos a taxa de queda por sexo, houve diferença estatística $(p=0,01)$. Este evento esteve presente em $35,4 \%$ dos pacientes com diagnóstico de osteoporose densitométrica. Dentre os participantes do estudo, $15(10,3 \%)$ relataram fratura, com predomínio no sexo feminino $(80 \%)$, porém sem diferença estatística. (Tabela 3)

Ao calcular o risco absoluto de fraturas osteoporóticas através da ferramenta FRAX clínico, sem inclusão de densitometria óssea, foi observado que toda a amostra teve média de 2,4 $\pm 2,3 \%$ para fraturas osteoporóticas de quadril em 10 anos, sendo que em homens a média foi de $1,8 \pm 1,3 \%$ e em mulheres a média foi de $2,7 \pm 2,5 \%$. Em relação ao risco de fratura osteoporótica maior em 10 anos, calculado para todos os participantes, a média foi de $5,8 \pm 4,0 \%$, onde o sexo masculino obteve média de $3,6 \pm 1,6 \%$ e o feminino média de $6,7 \pm 4,3 \%$. Houve diferença estatística entre os sexos e entre o risco de fratura de quadril e fratura maior quando avaliado os escores obtidos, entretanto quando avaliamos em relação ao número absoluto de indivíduos, não foi observada diferença estatística para risco de fratura maior (Tabela 4). 
Ao avaliar o risco absoluto de fratura de quadril e fratura maior em relação a faixa etária por meio da ferramenta FRAX, observou-se que na faixa etária de 65 a 69, 70 a 79 e 80 a 90, houve um, 21 e 16 pacientes, respectivamente, com risco de fratura de quadril $(\geq 3 \%)$. Apenas dois apresentaram risco de fratura maior $(\geq 20 \%)$, sendo observado na faixa etária de 70 a 79 anos e na de 80 a 90 anos. Observamos que o risco de fratura de quadril é mais elevado do que o risco de fratura maior, $p=0,0001$, nas faixas etárias de 70 a 79 anos e dos 80 aos 90 anos, porém não houve diferença estatística entres as faixas etárias estudadas para o risco de fratura maior e de quadril (Tabela 5).

Ao considerar os resultados obtidos a partir da ferramenta FRAX clínico para risco absoluto para fratura osteoporótica de quadril para todos os voluntários do estudo, dos $38(26,2 \%)$ que possuíam risco absoluto para fraturas de quadril, seis foram do sexo masculino e 32 do feminino. Dos dois participantes que apresentaram risco para fratura maior, todos eram do sexo feminino.

Quando avaliamos o risco de fratura maior e risco de fratura de quadril e classificamos por sexo e faixa etária observamos que em todas as faixas etárias estudadas o sexo feminino obteve maior escore por meio da ferramenta FRAX clínico, com diferença estatística, exceto para risco de fratura de quadril na faixa etária de 65 a 69 anos.

Em relação aos $31(21,4 \%)$ pacientes diagnosticados com osteoporose, 19 realizavam suplementação de cálcio e vitamina $D$, dois apenas de cálcio e 00 apenas de vitamina. Nenhum participante realizava tratamento farmacológico específico para osteoporose.

Tabela 3

Avaliação de episódio quedas e número de fratura em relação a faixa etária e sexo. $(n=145)$

\begin{tabular}{|c|c|c|c|c|c|}
\hline Faixa etária (a) & $\mathbf{n}$ & $\begin{array}{l}\text { Sexo } \\
F(n) \\
M(n)\end{array}$ & $\begin{array}{c}\text { Taxa de queda por } \\
\text { sexo }(\%)\end{array}$ & $\begin{array}{l}\text { Taxa de queda por } \\
\text { Faixa etária }(\%)\end{array}$ & Número de Fratura \\
\hline $65-69 a$ & 70 & $\begin{array}{l}\text { M (17) } \\
F(53)\end{array}$ & $\begin{array}{c}2(11,8) \\
21(39,6)\end{array}$ & 32,8 & $\begin{array}{l}0 \\
6\end{array}$ \\
\hline$p$ & - & - & 0,13 & - & 0,32 \\
\hline $70-79 a$ & 53 & $\begin{array}{l}M(17) \\
F(36)\end{array}$ & $\begin{array}{c}2(11,8) \\
12(33,3)\end{array}$ & 26,3 & $\begin{array}{l}3 \\
5\end{array}$ \\
\hline$p$ & - & - & 0,18 & - & 0,7 \\
\hline $80-90 a$ & 22 & $\begin{array}{l}M(9) \\
F(13)\end{array}$ & $\begin{array}{l}3(33,3) \\
6(46,2)\end{array}$ & 40,9 & $\begin{array}{l}0 \\
1\end{array}$ \\
\hline $\mathrm{p}$ & - & - & 0,67 & - & 1,0 \\
\hline Total & 145 & $\begin{array}{l}M(43) \\
F(102)\end{array}$ & $\begin{array}{c}7(16,3) \\
39(38,2)\end{array}$ & 31,7 & $\begin{array}{c}3 \\
12\end{array}$ \\
\hline $\mathrm{p}$ & - & - & 0,01 & - & 0,55 \\
\hline
\end{tabular}

a: anos; n: número; F: feminino; M: masculino; p: teste de Fisher

\section{Tabela 4}

Avaliação do risco absoluto de fratura de quadril e fratura maior em relação ao sexo por meio da ferramenta FRAX. $(n=145)$

\begin{tabular}{|c|c|c|c|c|}
\hline \multicolumn{2}{|c|}{$\begin{array}{c}\text { Risco de fratura de quadril } \\
X \pm D P\end{array}$} & \multicolumn{2}{|c|}{$\begin{array}{c}\text { Risco de fratura maior } \\
\qquad X \pm D P\end{array}$} & \multirow{2}{*}{$\begin{array}{c}\mathbf{p} \\
0,0001 a\end{array}$} \\
\hline Masculino & $1,8 \pm 1,3 \%$ & Masculino & $3,6 \pm 1,6 \%$ & \\
\hline Feminino & $2,7 \pm 2,5 \%$ & Feminino & $6,7 \pm 4,3 \%$ & $0,0001 \mathrm{a}$ \\
\hline Total & $2,4 \pm 2,3 \%$ & Total & $5,8 \pm 4,0 \%$ & $0,0001 a$ \\
\hline $\mathrm{p}$ & $0,04 a$ & $\mathrm{p}$ & $0,0001 \mathrm{a}$ & - \\
\hline
\end{tabular}


Tabela 4 (Continuação)

\begin{tabular}{|c|c|c|c|c|}
\hline \multicolumn{2}{|c|}{$\begin{array}{l}\text { Risco de fratura de quadril } \\
(\geq 3 \%)\end{array}$} & \multicolumn{2}{|c|}{$\begin{array}{c}\text { Risco de fratura Maior } \\
(\geq 20 \%)\end{array}$} & \multirow{2}{*}{$\frac{-}{0,02 \mathrm{~b}}$} \\
\hline Masculino & 6 & Masculino & 0 & \\
\hline Feminino & 32 & Feminino & 2 & $0,0001 b$ \\
\hline Total & 38 & Total & 2 & $0,0001 b$ \\
\hline $\mathrm{p}$ & $0,03 \mathrm{~b}$ & $\mathrm{p}$ & $0,55 \mathrm{~b}$ & - \\
\hline
\end{tabular}

X: Média; DP: desvio-padrão; a: Teste Mann Whitney; b: Teste de Fisher

\section{Tabela 5}

Avaliação do risco absoluto de fratura de quadril e fratura maior em relação a faixa etária por meio da ferramenta FRAX. $(n=145)$

\begin{tabular}{|c|c|c|c|c|}
\hline Faixa etária (a) & $\mathbf{n}$ & $\begin{array}{l}\text { Risco de fratura } \\
\text { Maior } \geq 20 \% \\
(X \pm D P)\end{array}$ & $\begin{array}{c}\text { Risco de fratura } \\
\text { De quadril } \geq 3 \% \\
N(X \pm D P)\end{array}$ & $\mathbf{p}$ \\
\hline $65-69 a$ & 70 & $0(3,9 \pm 1,6)$ & $1(1,2 \pm 0,6)$ & 1,0 a \\
\hline $70-79 a$ & 53 & $1(6,7 \pm 4,3)$ & $21 \quad(3,0 \pm 2,5)$ & $0,0001^{a}$ \\
\hline $80-90 a$ & 22 & $1(9,6 \pm 4,9)$ & $16(5,0 \pm 2,4)$ & $0,0001^{a}$ \\
\hline Total & 145 & 2 & 38 & $0,0001^{a}$ \\
\hline$P$ & & $0,8 b(0,0001) b$ & $0,7 \quad(0,0001) b$ & - \\
\hline
\end{tabular}

a: anos; n: número; DP: desvio-padrão; a: teste de Fisher; b: ANOVA

\section{DISCUSSÃO}

A osteoporose é uma das principais causas de mortalidade e morbidade na população idosa. A identificação de grupos com maior risco para o desenvolvimento desta patologia é bastante importante devido ao alto custo e dificuldade de tratamento para o sistema de saúde ${ }^{13}$.

Nesta casuística de 145 participantes, com idade média de 71 anos, 31 participantes $(21,4 \%)$ relataram apresentar o diagnóstico de osteoporose e, destes, 29 pertenciam ao sexo feminino. Tal fato se deve à menor massa óssea por unidade de volume de osso das mulheres em comparação aos homens, em todas as idades. Após a menopausa, os níveis do hormônio estrogênio estão reduzidos, o que gera maior ativação dos ciclos de remodelação óssea, com a fase de reabsorção predominando comparada à formação ${ }^{14}$.

Em um estudo feito por Rodrigues e Barros $(2016)^{14}$, também houve maior prevalência de osteoporose no sexo feminino. Dentre os 1419 integrantes do estudo, $841(59,3 \%)$ foram mulheres e $578(40,7 \%)$, homens. A prevalência de osteoporose no sexo feminino $(22,8 \%)$ foi bastante superior à do sexo masculino $(4,4 \%)$.
Os principais fatores de risco para osteoporose presentes em nosso estudo foram: sedentarismo $(53,1 \%)$, uso de corticoide $(18,6 \%)$, menopausa precoce $(15,9 \%)$, tabagismo $(11 \%)$, etilismo $(3,4 \%)$, fratura de quadril em pais $(1,4 \%)$, síndrome de má absorção $(1,4 \%)$ e uso de anticonvulsivante $(1,4 \%)$. Isso demonstra a importância da história e do exame físico minuciosos antes do início do tratamento para osteoporose, com o intuito de intervir nos fatores de risco modificáveis, como sedentarismo, tabagismo e etilismo ${ }^{1}$.

A média do índice de massa corporal em nossa casuística foi de $27,5 \mathrm{~kg} / \mathrm{m}^{2}$ (sobrepeso), este achado pode estar associado ao sedentarismo na população estudada. A ausência da prática de exercícios físicos está relacionada com perda da massa muscular e prejuízo no equilíbrio, o que contribui para o risco de quedas e fraturas. Entretanto, a indicação de qualquer atividade física depende do estado de saúde e capacidade funcional individual ${ }^{15}$.

Dentre os entrevistados do estudo, 86 $(59,3 \%)$ afirmaram já terem realizado DXA, com maior prevalência no sexo feminino (86\%). De acordo com o Ministério da Saúde $(2014)^{13}$, 
independente dos fatores riscos, a DXA está indicada para mulher com idade igual ou superior a 65 anos e homens com idade igual ou superior a 70 anos. Considerando que a casuística apresentou 102 mulheres e 43 homens, sendo que 26 homens apresentaram idade igual ou superior a 70 anos, pelo menos 128 participantes já deveriam ter sido submetidos a DXA. A baixa DMO, além de poder ser usada como método diagnóstico, é preditor de fraturas. Para cada diminuição de um desvio padrão, há um aumento de duas a três vezes no risco de fraturas ${ }^{1}$.

As quedas em idosos geralmente predispõe ao medo do cair e à limitação da prática de atividades, o que afeta a saúde e eleva o risco de institucionalização e, consequentemente, de trazer problemas físicos e psicológicos, além de elevar os custos com os cuidados da saúde ${ }^{16}$. Neste estudo, observou-se que $46(31,7 \%)$ pacientes relataram já ter sofrido pelo menos um episódio de queda, sendo 39 do sexo feminino e sete do sexo masculino. A faixa etária entre 65 e 69 anos apresentou maior frequência de quedas, com 23 relatos. Destes, 21 foram do sexo feminino e, apenas dois, do sexo masculino.

Lima et al. (2017) ${ }^{17}$, numa pesquisa com 418 idosos, 63,8\% dos participantes relataram ter apresentado pelo menos uma queda no último ano, porcentagem mais elevada comparada a este presente estudo (31,7\%). Entretanto, houve diferença na faixa etária de maior ocorrência de quedas. No estudo feito por Lima et al., dos $63,8 \%$ que relataram pelo menos um episódio de queda, $41,2 \%$ possuíam idade superior a 80 anos.

No estudo de Souza et al. (2019) ${ }^{16}, 40 \%$ dos participantes do grupo sem o diagnóstico de osteoporose, ao serem questionados sobre as formas de prevenção da doença, relataram não apresentar conhecimento sobre osteoporose. Tendo em vista que a população não tem conhecimento referente a osteoporose é bem provável que o conhecimento referente a prevenção de quedas seja escasso. Diante da associação entre osteoporose, queda e fratura, é importante a implementação de medidas que possuam como principal objetivo minimizar o risco de quedas, como: apoios e tapetes antiderrapantes no banheiro; melhoria da luminosidade; cuidados com escadas e degraus; correção de déficits visuais.
Em idoso, a fratura óssea é uma das principais causas de hospitalização. Após uma fratura, o indivíduo acometido frequentemente torna-se funcionalmente depende, seja durante uma reabilitação imediata ou a longo prazo. Além disso, cerca de $50 \%$ dos pacientes com fratura não recuperam sua mobilidade prévia. No Reino Unido, 536 mil fraturas/ ano estão associadas com a osteoporose e o custo anual ultrapassa 4,4 bilhões de libras. Nos Estados Unidos, o custo anual estimado é de 20 bilhões de dólares ${ }^{18}$. No presente estudo, 15 (10,3\%) pacientes relataram já apresentado fratura. Desses, 12 pertenciam ao sexo feminino e apenas três do sexo masculino. A maior ocorrência de fraturas foi na faixa etária de 70 a 79 anos.

O FRAX é um algoritmo utilizado para estimar a probabilidade individualizada de fraturas do quadril $(\geq 3 \%)$ e de fratura maior $(\geq 20 \%)$, que inclui fratura de coluna vertebral, antebraço, ou úmero, em 10 anos. Está disponível em 63 países e em 32 idiomas, alcançando $79 \%$ da população mundial. Foi a primeira ferramenta a fornecer um modelo de previsão de fratura específica no Brasil. Inclui oito fatores de risco clínico para estimar o risco de fratura em 10 anos: fratura anterior por fragilidade, fratura de quadris dos pais, tabagismo, uso sistêmico de glicocorticoide, ingestão excessiva de álcool, índice de massa corporal, artrite reumatoide e outras causas de osteoporose secundária; além da idade, sexo e DMO no colo do fêmur (opcional) ${ }^{19}$.

Nesta casuística, $40(27,6 \%)$ pacientes apresentaram risco de fratura, sendo $38(26,2 \%)$ casos por risco de fratura de quadril $\geq 3 \%$. Destes, 21 possuíam idade entre 70 a 79 anos (sendo 19 do sexo feminino e dois do masculino), 16 entre 80 e 90 anos (sendo 13 do sexo feminino e três do masculino) e apenas um entre 65 a 69 anos (sendo um do sexo masculino e nenhum do feminino). 0 risco de fratura osteoporótica maior $\geq 20 \%$ ocorreu em apenas dois pacientes do sexo feminino, sendo um da faixa etária de 70 a 79 anos e outro da faixa etária de 80 a 90 anos.

De acordo com o Ministério da Saúde $(2014)^{13}$, o tratamento da osteoporose consiste em condutas não medicamentosas e medicamentosas. Dentre as não medicamentosas, incluem: prática de exercício físico, medidas de prevenção de quedas (medidas de segurança ambiental; avaliação de problemas neurológicos, correção de 
distúrbios visuais e auditivos) e desencorajar o tabagismo e o etilismo. Com relação a suplementação de nutrientes, o cálcio e a vitamina $D$ são os mais importantes. Portanto, a suplementação destes nutrientes é o tratamento padrão na prevenção de fraturas e deve-se garantir a ingestão diária mínima em todos os casos. Recomenda-se a ingestão de 1.200 a $1.500 \mathrm{mg}$ de cálcio elementar por dia e 800-1.000 UI de vitamina $D$ para adultos com 50 anos ou mais.

Os principais medicamentos que diminuem fraturas osteoporóticas são os bifosfonatos e os orais são os medicamentos de escolha para o tratamento. Apesar de não existir evidência de superioridade dentre os tipos de bifosfonatos, o alendronato de sódio ou risedronato de sódio são os de escolha. Neste presente estudo, dos 31 pacientes diagnosticados com osteoporose, 19 faziam suplementação de cálcio e vitamina $D$, dois apenas de Cálcio, um apenas de Vitamina $D$, nenhum fazia tratamento farmacológico específico e nove não faziam suplementação e tratamento farmacológico específico. Isso demonstra que mais pacientes deveriam realizar o tratamento adequado da osteoporose com o intuito de evitar complicações graves, como novas fraturas e deformidades secundárias, dor crônica e perda da independência, conforme também observado em outros estudos ${ }^{20}$.

\section{CONCLUSÃO}

Por meio do nosso estudo podemos observar que a osteoporose, embora seja uma doença com alta prevalência e com grandes implicações clínicas, ainda é subdiagnosticada e subtratada em nosso meio. A frequência de quedas na população idosa é um achado frequente e com consequências graves, como fraturas.

Nossos dados também demonstram que os fatores de risco para osteoporose são prevalentes na população idosa e a associação deles aumenta o risco de fratura, como evidenciado pela aplicação da ferramenta FRAX clínico. Evidenciamos que a mesma tem melhor capacidade de identificar risco de fratura de quadril, quando comparada ao risco de fratura maior, além de demonstrar que o sexo feminino e indivíduos mais idosos tem maiores risco de fratura.

\section{REFERÊNCIAS}

1. Radominski SC, Bernardo $W$, Paula AP, Albergaria $\mathrm{B}-\mathrm{H}$, Moreira C, Fernandes $\mathrm{CE}$, et al. Diretrizes brasileiras para o diagnóstico e tratamento da osteoporose em mulheres na pós-menopausa. Rev Bras Reumatol. 2017;57(S2):S452-S466. Disponível em: https://www.sciencedirect.com/science/article/pii/ s048250041730178X

2. Costa ALD, Silva MACN, Brito LMO, Nascimento ACB, Barbosa MCL, Batista JE, et al. Osteoporose na atenção primária: uma oportunidade para abordar os fatores de risco. Rev Bras Reumatol. 2016;56(2):111-6. Disponível em: https://www.sciencedirect.com/science/ article/pii/S0482500415000704?via\%3Dihub

3. Silva ACV, Rosa MI, Fernandes B, Lumertz S, Diniz RM, Damiani MEFR. Fatores associados à osteopenia e osteoporose em mulheres submetidas à densitometria óssea. Rev Bras Reumatol. 2015;55(3):223-8. Disponível em: https://www.sciencedirect.com/science/article/pii/ S0482500414002095?via\%3Dihub

4. Lane NE. Epidemiology, etiology, and diagnosis of osteoporosis. Am J Obstet Gynecol. 2006:194(2, Supplement):S3S11. Disponível em: https://www.sciencedirect.com/ science/article/pii/S0002937805013700?via\%3Dihub

5. Baccaro LFC, Paiva LC, Pinto Neto AM. Osteoporose. Rev Bras Med. 2014;71(3):48-5.

6. Cosman F, Beur SJ, LeBoff MS, Lewiecki EM, Tanner $B$, Randall $S$, et al. Clinician's guide to prevention and treatment of osteoporosis. Osteoporos Int. 2014;25(10):2359-81.

7. Souza BGS, Carvalho LGVA, Oliveira LFMM, Ferreira AG, Amaral RCS, Oliveira VM. Profilaxia primária e secundária de fraturas osteoporóticas: avaliação de uma coorte prospectiva. Rev Bras Ortop. 2017;52(5):538-43. Disponível: https://www.sciencedirect.com/science/ article/pii/S0102361616301023

8. MacLean C, Alexander A, Carter J, Chen S, Desai SB, Grossman J, et al. Comparative effectiveness of treatments to prevent fractures in men and women with low bone density or osteoporosis. Rockville (MD): Agency for Healthcare Research and Quality (US); 2007 Dec. Report No.: 08-EHC008-EF. Disponível em: https:// www.ncbi.nlm.nih.gov/books/NBK43160/

9. Frazão $P$, Naveira $M$. Prevalência de osteoporose: uma revisão crítica. Rev Bras Epidemiol. 2006;9(2):206-14. Disponível em: https://www.scielo.br/scielo.php?pi$\mathrm{d}=$ S1415-790X2006000200007\&script=sci_abstract\&tlng=pt

10. Bernabei R, Martone AM, Ortolani E, Landi F, Marzetti E. Screening, diagnosis and treatment of osteoporosis: a brief review. Clin Cases Miner Bone Metab. 2014;11(3):201-7.

11. Bastos-Silva Y, Aguiar LB, Pinto-Neto AM, Baccaro LF, Costa-Paiva L. Correlation between osteoporotic fracture risk in Brazilian postmenopausal women calculated 
using the FRAX with and without the inclusion of bone densitometry data. Arch Osteoporos. 2016;11:16.

12. Sousa CJ, Oliveira MLC. FRAX Tool in Brazil: an integrative literature review following validation. Rev Bras Geriatr Gerontol. 2018;21(1):108-15. Disponível em: https://www.scielo.br/scielo.php?script=sci_arttext\&pi$\mathrm{d}=$ S1809-98232018000100108

13. Protocolo Clínico e Diretrizes Terapêuticas da Osteoporose. PORTARIA No 224, DE 26 DE MARÇO DE 2014. Ministério da Saúde.

14. Rodrigues IG, Barros MBA. Osteoporose autorreferida em população idosa: pesquisa de base populacional no município de Campinas, São Paulo. Rev Bras Epidemiol. 2016;19(2):294-306. Disponível em: https://www.scielo.br/scielo.php?script=sci_arttext\&pid=S1415-790X2016000200294

15. Loures MAR, Zerbini CAF, Danowski JS, Pereira RMR, Moreira C, Paula AP, et al. Guidelines of the Brazilian Society of Rheumatology for the diagnosis and treatment of osteoporosis in men. Rev Bras Reumatol. 2017;57(supl.2):s497-514. Disponível em: https:// www.scielo.br/scielo.php?script $=$ sci_arttext\&pi$d=$ S0482-50042017000800009

16. Souza Júnior EÁ, Ferreira NF, Lopes PEC, Torres MS, Baumfeld DS, Andrade MAP de. Significado e considerações sobre a osteoporose por mulheres com e sem diagnóstico da doença. ABCS Health Sci [Internet]. 2019;44(1). Disponível em: https://www.portalnepas. org.br/abcshs/article/view/1116

17. Lima AP, Lini EV, Dellani MP, Portella MR, Doring $M$. Prevalência e fatores associados às quedas em idosos de Estação-RS: estudo transversal de base populacional. Cad Saúde Colet. 2017;25(4):436-42. Disponível em: https://www.scielo.br/scielo.php?pi$\mathrm{d}=$ S1414-462X2017000400436\&script=sci_abstract\&tlng $=p t$

18. Rocha VM, Gaspar HA, Oliveira CF de, Rocha VM, Gaspar HA, Oliveira CF. Fracture risk assessment in home care patients using the FRAX® tool. Einstein (São Paulo) [Internet]. 2018;16(3). Disponível em: https://www. scielo.br/scielo.php?script $=$ sci_abstract\&pid $=$ S1679$45082018000300206 \& \mathrm{lng}=$ en $\& \mathrm{nrm}=$ iso\&tlng $=$ en

19. Albergaria $\mathrm{BH}$, Paula FJA. The Algorhytm: FRAX Brazil. Rev Bras Ginecol Obstet. 2019;41(8):467-8. Disponível em: https://www.scielo.br/scielo.php?script=sci_arttext\&pid=S0100-72032019000800467

20. Zamboni C, Carvalho MS, Pires EA, Durigan JR, Fucs PMMB, Mercadante MT. Are traumatologists treating osteoporosis to prevent new fractures in Brazil? Acta Ortop Bras. 2018;26(6):384-7. Disponível em: https://www.scielo.br/scielo.php?script=sci_arttext\&pi$\mathrm{d}=\mathrm{S} 1413-78522018000600384$ 